\title{
Mother Behavior
} Q-Set (MBQS) Dimensionalidad del Q-set dél comportamiento materno en muestras de México, Colombia y Uruguay

Mother Behavior Q-Set (MBQS): Dimensionality of the Mother Behavior Q-Set in samples from Mexico, Colombia and Uruguay.

(c) (i) (2) (2)

Fernando Salinas-Quiroz Antonio Martínez Pineda Sandra Juliana Plata Contreras Paola Elizabeth Silva Cabrera Verónica Cambón Mihalfi Wendolyn Cortes Rojas
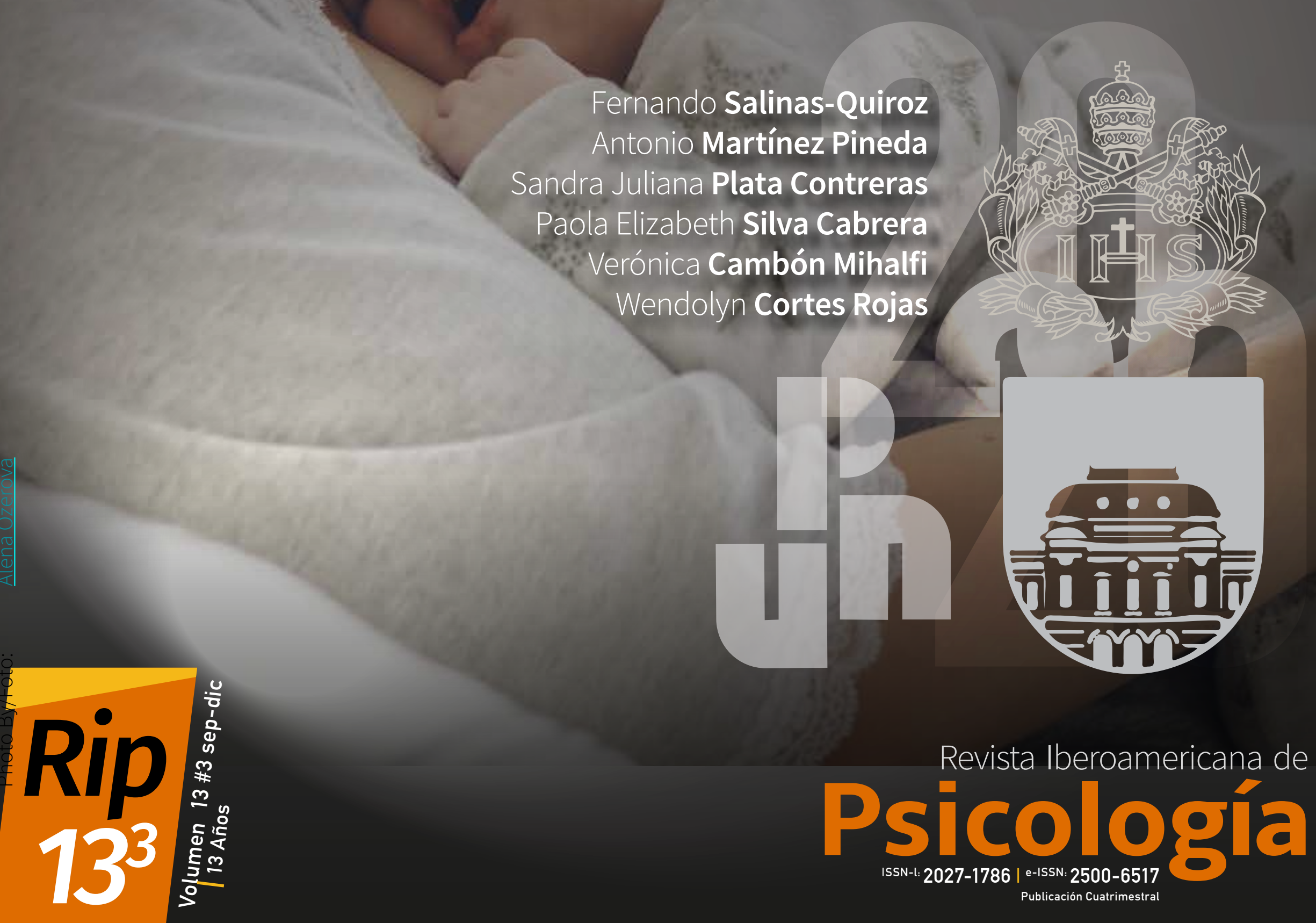
Revista Iberoamericana de

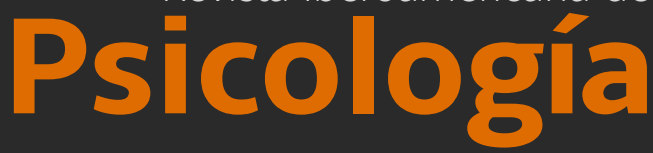

ISSN-I: 2027-1786 | e-ISSN: 2500-6517 Publicación Cuatrimestral

ID: 2027-1786.rip.13307

Title: $\quad$ Mother Behavior Q-Set (MBQS): Dimensionality of the Mother Behavior Q-Set in samples from Mexico, Colombia and Uruguay.

Título: $\quad$ Mother Behavior Q-Set (MBQS)

Subtítulo: Dimensionalidad del q-set del comportamiento materno en muestras de méxico, colombia y uruguay

Alt Title / Título alternativo:

[en]: $\quad$ Mother Behavior Q-Set (MBQS): Dimensionality of the Q-set of maternal behavior in samples from Mexico, Colombia and Uruguay

[es]: $\quad$ Mother Behavior Q-Set (MBQS): Dimensionalidad del Q-set del comportamiento materno en muestras de México, Colombia y Uruguay

Author (s) / Autor (es):

Salinas-Quiroz, Martínez Pineda, Plata Contreras, Silva Cabrera, Cambón Mihalfi, \& Cortes Rojas

Keywords / Palabras Clave:

[en]: maternal sensitivity; Q-methodology; Q-sort; factor analysis; principal components analysis; $Q$ analysis

[es]: $\quad$ sensibilidad materna; metodología Q; Q-sort; análisis factorial confirmatorio; análisis de componentes principales; análisis Q

Submited: 2021-01-13

Acepted: 2020-09-01

\section{Resumen}

La sensibilidad se refiere a la capacidad del interpretarlas correctamente y responder a ellas de manera contingente y apropiada. El Q Set del Comportamiento Materno (MBQS) fue creado a partir de descripciones teóricas y empíricas de la sensibilidad. Los Q-sorts se asocian con la metodología cuantitativa

debido a que asignan puntajes; además, combinan la metodología cualitativa al hacer un estudio sistemático de la subjetividad. Son pocos los artículos que se ocupan de explorar el análisis, la validez interna, la confiabilidad o la replicabilidad externa de los Q-sorts. Usualmente los datos se reducen por medio de un análisis de componentes principales o análisis factorial. Sin embargo, en los Q-sorts

se correlacionan los participantes para

correlacionar las variables. El objetivo de la presente investigación fue encontrar evidencia respecto a la validez basada en la estructura interna del MBQS con el apoyo del software R y el paquete qmethod. Colaboraron 37 díadas mexicanas mamá-bebés, 35 colombianas y 24 uruguayas. En el 2002, Posada y colaboradores propusieron siete escalas. Las escalas en las

que nuestro modelo factorial presentó un buen ajuste son las que tienen menos ítems, lo cual puede ser indicativo de que la falta de ajuste tiene que ver con la identificación del

modelo debido a que se cuenta con pocas observaciones. Consideramos que tanto el uso del MBQS para evaluar la sensibilidad como del paquete qmethod para realizar el análisis resulta novedoso, relevante y pertinente. cuidador para percibir las señales del niño, dilucidar las relaciones entre ellos en lugar de

\section{Abstract}

Sensitivity refers to the caregiver's ability to perceive infant's cues, interpret them correctly, and respond to them in a contingent and appropriate way. The Maternal Behavior Q Set (MBQS) was created from theoretical and empirical descriptions of sensitivity.

Q-methodology is associated with the quantitative methodology because it assigns scores; in addition, it combines qualitative methodology by making a systematic study of subjectivity. Few papers are concerned with exploring the analysis, internal validity,

reliability, or external replicability of Q

studies. Usually analyses reduce data to a few summarizing factors, based on principal component analysis or centroid factor analysis.

Q-factor analysis method was developed especially for q-sorted data; it is a by-person

factor analysis which is used to identify groups of participants who make sense of a pool of items in comparable way. The aim of this study was to find evidence regarding the validity based on the internal structure of the MBQS using the R software and the qmethod package. Thirty-seven Mexican, 35 Colombian and 24 Uruguayan mother-baby dyads collaborated. In 2002, Posada et al. proposed seven scales. The scales in which our factorial model presented a good fit are those with the fewest items, which may be indicative that the

lack of fit has to do with the identification of the model because there are few observations.

We consider that both the use of the MBQS to assess sensitivity and the qmethod package to perform the analysis is novel, relevant and pertinent.

\section{Citar como:}

Salinas-Quiroz, F., Martínez Pineda, A., Plata Contreras, S. J., Silva Cabrera, P. E., Cambón Mihalfi, V., \& Cortes Rojas, W. (2020). Mother Behavior Q-Set (MBQS): Dimensionalidad del q-set del comportamiento materno en muestras de méxico, colombia y uruguay. Revista Iberoamericana de Psicología, 13 (3), [pg|n]-[pgOut]. Obtenido de: https://reviberopsicologia.ibero.edu.co/article/ view/1955

DrFernando Salinas-Quiroz,Ph.D MA PsiLic
Research ID: $\quad \underline{\text { D-8247-2013 }}$
ORCID: $\quad \underline{000-0002-1257-6379}$
Source I Filiacion:
Universidad Pedagógica Nacional (México)
BIO:
Investigador Nacional, Sistema Nacional de Investigadores.
Vicepresidente de la Red Iberoamericana de Apego.
City I Ciudad:
Ciudad de México [mx]
e-mail:
fsalinas@upn.mx
Paola Elizabeth Silva Cabrera, MA PsiLic
ORCID:
Source I Filiacion:
Universidad de la República
BIO:
Enseñanza, Extensión e Investigación en el marco del Instituto
de Psicología, Educación y Desarrollo Humano
City I Ciudad:
Montevideo [uy]
e-mail:
psilva@psico.edu.uy\}

Antonio Martínez Pineda, PsiLic

AutorID:

ORCID:

Source I Filiacion

Centro Nacional de Evaluación para la Educación Superior BIO:

Jefe del Departamento de Calidad Técnica en la Dirección de Investigación, Calidad e Innovación del Ceneval,

City I Ciudad:

Ciudad de México $[\mathrm{mx}]$

e-mail:

antonio.martinez@ceneval.edu.mx

C, MA PsiLic
ORCID: $\quad$ 0000-0003-4030-5987
Source I Filiacion:
Universidad de la República
BIO:
Docente Universitario e investigadora. Consultora en Política
Pública de Primera Infancia.
City I Ciudad:
Montevideo [uy]
e-mail:
vcambon@psico.edu.uy

Sandra Juliana Plata Contreras, MA Psi sp

ORCID: $\quad$ 0000-0001-6353-2627

Source I Filiacion:

Pontificia Universidad Javeriana - Bogotá, Colombia BIO:

Profesora Asistente Tiempo Completo. Coordinadora

Práctica Familias, Vinculos y Violencias. Carrera de Psicologia. Investigadora Asociada - Minciencias. Colombia.

City I Ciudad:

Bogotá DC $[\mathrm{CO}]$

e-mail:

sandra.plata@javeriana.edu.co

\section{Wendolyn Cortes Rojas, PsiLic}

ORCID: OSource I Filiacion:

Universidad Pedagógica Nacional (México)

BIO:

Asistente de investigación

City I Ciudad:

Ciudad de México $[\mathrm{mx}]$

e-mail:

ps.nicolas.didier@gmail.com 


\section{Mother Behavior Q-Set (MBQS) Dimensionalidad del Q-set del
comportamiento materno en muestras de
México, Colombia y Uruguay}

Mother Behavior Q-Set (MBQS): Dimensionality of the Mother Behavior Q-Set in samples from Mexico, Colombia and Uruguay.

Fernando Salinas-Quiroz

Antonio Martínez Pineda

Sandra Juliana Plata Contreras

Paola Elizabeth Silva Cabrera

Verónica Cambón Mihalfi

Wendolyn Cortes Rojas

Los vínculos de apego cuidador-bebé surgen de la interacción: si se les da la oportunidad, todos los bebés se apegan a uno o más cuidadores, lo que se conoce como la hipótesis de la universalidad (van IJzendoorn y Sagi-Schwartz, 2008). Un aspecto central de la teoría de Mary Ainsworth y John Bowlby tiene que ver con el papel del cuidador principal como una base segura desde la cual un bebé puede organizar su comportamiento, obtener seguridad, explorar y aprender sobre el medio ambiente (e.g., Ainsworth, 1967; Ainsworth, Blehar, Waters y Walls, 1978; Bowlby, 1969/1982, 1988). La sensibilidad se refiere a la capacidad del cuidador para percibir las señales del niño, interpretarlas correctamente y responder a ellas de manera contingente y apropiada en las circunstancias en las que se encuentra la díada (Ainsworth, Bell y Stayton, 1974).

Una madre sensible centra su atención en las necesidades físicas y emocionales de su hijo o hija y es capaz de ver las cosas desde el punto de vista del niño o niña. Asimismo, sincroniza las actividades de su hijo o hija con las propias, negocia ante conflictos y se ajusta a los estados emocionales, momento evolutivo y particularidades del sujeto menor (Salinas-Quiroz y Posada, 2015, p.1056). 


\section{Mother Behavior Q-Set (MBQS)}

\section{Dimensionalidad del q-set del comportamiento materno en muestras de méxico, colombia y uruguay}

Ni la sensibilidad del cuidador, ni la seguridad del niño son rasgos de personalidad: ambas son construcciones diádicas que ocurren en el contexto de una relación de apego específica (Posada, Carbonell, Alzate y Plata, 2004) y que hablan de la calidad de la interacción (Duarte-Rico, García Ramírez, Rodríguez Cruz y Bermúdez Jaimes, 2016). En otras palabras, se trata de sistemas complementarios que se codeterminan entre sí (Posada y Waters, 2018). La hipótesis de la sensibilidad sugiere que el vínculo entre sensibilidad y seguridad se mantiene en diferentes contextos sociales, situaciones y culturas. A la fecha, numerosos metaanálisis han confirmado esto (Cadman, Diamond y Fearon, 2018; De Wolff y van IJzendoorn, 1997; Verhage et al., 2016; Zeegers, Colonnesi, Stams y Meins, 2017), con rs que van entre .24 y .39 (Cadman et al., 2008; Zeegers et al., 2017), y muestran que la sensibilidad tiene una conexión causal con la seguridad del apego infantil (Bakermans-Kranenburg, van ljzendoorn y Juffer, 2003).

Desafortunadamente, la mayoría de las investigaciones se han realizado en países occidentales industrializados (Posada et al., 2016). Dentro de las principales diferencias entre las familias anglosajonas y las familias latinoamericanas es que las últimas han sido descritas como más socialmente orientadas; en otras palabras, menos individualistas y con una tendencia a tener actitudes de crianza que enfatizan la interdependencia en lugar de la independencia (Posada et al., 2016). De hecho, Fourment, Nóblega, Conde, Nuñez del Prado y Mesman (2020) recientemente encontraron que madres peruanas de comunidades andinas y amazónicas fueron altamente sensibles y particularmente hábiles para realizar múltiples tareas a la vez (i.e. combinar sus quehaceres domésticos y trabajo en el campo al tiempo que respondían sensiblemente a sus hijos), además de que la presencia de múltiples cuidadores aseguraba que los niños estuvieran atendidos cuando las madres no podían hacerles caso, lo que reafirma lo anteriormente expuesto sobre la orientación social que distingue nuestros países latinoamericanos.

\section{Q-Set del Comportamiento Materno (MBQS)}

La sensibilidad ha sido mayormente evaluada mediante observaciones estructuradas y no-estructuradas en el laboratorio por períodos cortos de tiempo (Ainsworth, 1973; Atkinson et al., 2000; De Wolff y van ljzendoorn, 1997; Nievar y Becker, 2007). El Q Set del Comportamiento Materno -MBQS por sus siglas en inglés- fue diseñado por David Pederson y Greg Moran en el año de 1995 a partir de descripciones teóricas y empíricas de la conducta materna, teniendo en cuenta el constructo de sensibilidad planteado por Ainsworth y colaboradores (Salinas-Quiroz y Posada, 2015).

Los 90 ítems del MBQS describen un amplio rango de conductas de la madre en el contexto natural de cuidado, tales como sus prácticas de crianza; la organización del ambiente en función de las necesidades del niño; las interacciones durante la alimentación; la sensibilidad al estado de ánimo del menor de edad; específicamente la habilidad de la madre para reconocer y detectar las señales comunicativas del niño de forma pronta, oportuna y de acuerdo con sus necesidades (Carbonell, Plata, y Alzate, 2006). Los ítems del Q-Set de Comportamiento Materno se diseñaron explícitamente para distinguir el comportamiento materno sensible del no sensible (Pederson y Moran, 1995a).

El MBQS se asocia con la metodología cuantitativa debido a que asigna puntajes; además, combina las fuerzas y metodología del individuo investigador cualitativo, permitiendo hacer un estudio sistemático de la subjetividad. Cuenta con tres componentes: 1) procedimientos para desarrollar conjuntos de reactivos descriptivos para los cuales se asignan ciertos puntajes; 2) procedimientos para asignar puntajes a los reactivos, al acomodarlos dentro de un rango de ordenamiento, de lo más característico a lo menos característico del individuo participante evaluado, y; 3) una amplia variedad de procedimientos para la reducción y el análisis de los datos. Se trata de un método psicométrico que asigna calificaciones a un extenso número de reactivos (Salinas-Quiroz y Posada, 2015, pp.1056-1057).

El MBQS cuenta con 90 ítems: cada uno contiene un título y una descripción específica de la conducta a observar en la madre (Pederson y Moran, 1995b). El uso del MBQS asegura que distintos observadores evalúen el mismo contenido para describir a cada una de ellas.

Se les solicita a las personas evaluadoras que clasifiquen los reactivos en nueve categorías, buscando caracterizar y describir el comportamiento del sujeto cuidador evaluado. Los reactivos más característicos deben ubicarse en los lugares más altos -categorías 7 a 9-, y los menos característicos en las posiciones más bajas -categorías 1 a 3-; los que no caracterizan al cuidador o cuidadora, o no se observaron, se clasifican en el centro de la distribución-categorías 4 a 6-. Después de ajustar el número de reactivos en cada categoría, la distribución final debe ser de 10 en cada una de las nueve categorías y el puntaje de cada reactivo corresponde al número de la pila en que se ubicó. Una vez que cada evaluador o evaluadora finaliza su ordenamiento, se les pide que negocien con su pareja de observación aquellos reactivos con una diferencia en la categorización $\leq 3$ puntos. Tras discutir y modificar los desacuerdos, tanto el ordenamiento individual, como el negociado, se procesan... para establecer la confiabilidad interobservadores y obtener el puntaje de sensibilidad del individuo cuidador; este equivale a la correlación de la descripción de cada cuidador o cuidadora evaluado con el criterio teórico de un sujeto cuidador prototípicamente sensible (Salinas-Quiroz y Posada, 2015, p.1057).

En la siguiente figura se presenta la distribución de los ítems que supone el MBQS:

Figura 1. Distribución de los 90 ítems del MBQS

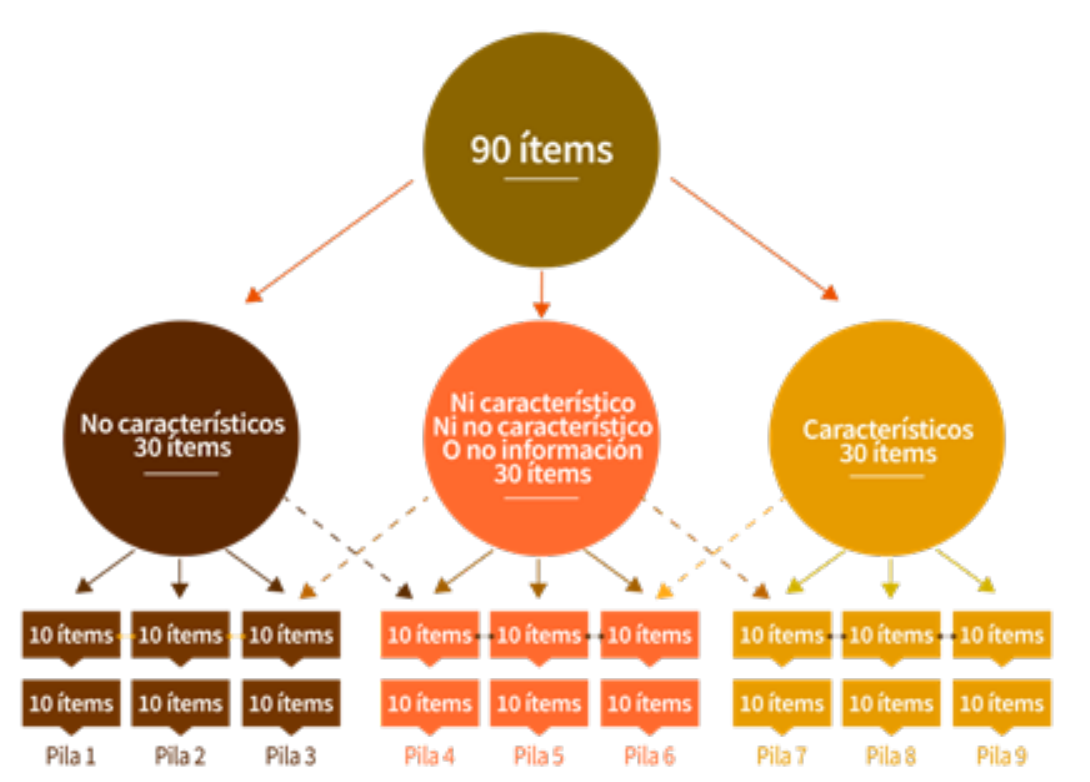

Fuente: elaboración propia

Se recomienda que dos observadores independientes realicen dos visitas distintas de entre hora y media y dos horas de duración cada una, para describir la conducta de la madre con el apoyo del MBQS (Posada y Waters, 2014). Si bien requiere de un exhaustivo entrenamiento y supervisión, el MBQS resulta idóneo para evaluar el comportamiento de las madres en contextos naturalísticos. 


\section{Dominios del MBQS}

En el año 2002, Germán Posada y colaboradores compararon la calidad del cuidado materno y la seguridad de los niños en 60 díadas estadounidenses y 61 colombianas (Posada et al., 2002). Posteriormente, identificaron dominios del cuidado materno característicos de las díadas de cada país, para lo cual realizaron un análisis factorial exploratorio (análisis de componentes principales) en cada muestra utilizando los 90 ítems del MBQS. Una vez que identificaron tales dominios, los compararon y posteriormente los analizaron en relación con la seguridad. Los resultados del análisis factorial exploratorio revelaron siete factores interpretables en cada muestra. Seis factores eran comunes a ambos contextos y dos no (un factor era específico para la muestra de Denver y otro para la muestra de Bogotá).

Las escalas se crearon sobre la base de las cargas de los elementos en cada factor. Los ítems que cargaban en más de un factor fueron asignados a una escala particular de acuerdo con consideraciones conceptuales. Se realizaron análisis de consistencia interna en cada escala. Se eliminaron los ítems que no contribuían a la confiabilidad de la escala y que tenían una correlación ítem-total de menos de .40. En seguida, las escalas obtenidas se relacionaron con todos los ítems del Q set. Los elementos que permanecieron en la escala fueron aquellos que tenían la mayor correlación con dicha escala en particular. Además, se incluyeron los ítems que originalmente no estaban colocados en la escala si: a) tenían una correlación de al menos .40; b) contribuían a la consistencia interna de una escala en particular, y; c) su contenido se ajustaba al de los otros ítems de la escala.

El primer factor para cada muestra contenía ítems que claramente pertenecían a las dimensiones de sensibilidad y accesibilidad identificadas por Ainsworth et al (1978). Así, de forma conceptual, subdividieron este factor en dos escalas: Respuesta Sensible y Accesibilidad. Asimismo, en la muestra de Bogotá, un factor adicional contenía ítems que pertenecían conceptualmente a dos escalas diferentes: Aceptación del bebé y Activa-Animada. Estos dos factores surgieron como separados en la muestra de Denver. Luego, procedieron a subdividir ese factor en dos en la muestra de Bogotá. Cada escala se nombró en función del contenido de los ítems incluidos en la misma.

Se identificaron los siguientes dominios en ambas muestras: (a) Respuesta sensible hace alusión justamente a la contestación de la madre ante las comunicaciones y señales del niño (e.g. Ítem 2: "No se da cuenta o es insensible a las señales de ansiedad del bebé"): (b) Accesibilidad, se refiere a la habilidad de la madre para tener en cuenta al niño pese a que compita con otras demandas (e.g. Ítem 10: "Saluda al bebé cuando entra a la habitación”); (c) Aceptación del bebé, que se refleja en el tono emocional positivo de la madre en sus interacciones con hijo (e.g. Ítem 13: "Se irrita con las demandas del bebé"); y (d) Interferencia, describe la incapacidad de la madre para cooperar con el comportamiento que presenta el niño (e.g. Ítem 59: "Ruda o intrusa en la interacción con el bebé"). Estos primeros cuatro dominios son paralelos a los propuestos por Ainsworth (Ainsworth et al., 1974; Ainsworth et al., 1978), lo cual no sorprende, pues el MBQS se construyó para reflejar su conceptualización sobre el comportamiento del cuidador.

Además, identificaron los siguientes dos dominios en ambas muestras: (e) Activa-Animada que indica la participación de la madre en su interacción con el niño (e.g. Ítem 34: "Busca interacciones frente a frente"), y ( $f$ ) Crea un ambiente interesante, se refiere al comportamiento materno relacionado con organizar un ambiente estimulante y apropiado para el niño (e.g. Ítem 18: "Organiza el ambiente considerando las necesidades del bebé y las suyas también”).
Finalmente, se identificó un dominio específico de la muestra para Denver y otro para Bogotá. El dominio específico para las participantes colombianas fue: (g) Preocupación por la apariencia física, el cual hace referencia al comportamiento de la madre que indica el interés en que el niño mantenga una buena presencia física (e.g. Ítem 50: "Algunas veces interfiere con una actividad apropiada del bebé si es posible que se bata o ensucie").

Posada et al. (2002) citan a Block (1978) arguyendo que los ítems en los Q-sorts no pueden ser independientes entre sí, pero que con un instrumento con alrededor de 100 ítems esta dependencia puede ser insignificante; no obstante, la identificación del modelo puede darse por la interdependencia entre los ítems o porque el número de parámetros a estimar es mayor (o similar) al número de observaciones en la muestra. Si bien consideramos que se trata de una propuesta sumamente valiosa, lo que se reporta en dicho artículo es un análisis de componentes principales -y no un análisis factorial-, por lo que desconocemos cuántos componentes obtuvieron en el mismo, pues reportaron que siete fueron interpretables.

Por otro lado, en el año 2017 Salman-Engin, Aran, Karagöbek y Sümer, evaluaron a 112 madres en Turquía con el apoyo del MBQS. A diferencia del estudio pionero de Posada et al. (2002), este equipo de investigación utilizó el método de análisis del factor Q para revelar los perfiles de cuidado materno. El análisis del factor Q es un análisis factorial por persona que se utiliza para identificar a grupos de participantes que dan sentido a un conjunto de ítems de manera comparable. En su estudio, las madres fueron tratadas como variables y los ítems del MBQS como casos con el apoyo del software R (R Core Team, 2020) y el paquete "qmethod" (Zabala, 2014). Encontraron dos perfiles de madres con una correlación de r=.53: aquellas Responsivas/cálidas y otras tantas Indiferentes/distantes.

\section{Análisis de la información recolectada en los Q-sorts}

En las últimas tres décadas, el proceso analítico ha recibido poca atención, pese a que se podría extraer más información relevante de los datos haciendo uso de los recientes avances estadísticos y computacionales. Son pocos los artículos que se ocupan de explorar el análisis, la validez interna, la confiabilidad o la replicabilidad externa de los estudios Q (Zabala y Pascual, 2016). El proceso analítico reduce los datos basados en análisis de componentes principales (PCA por sus siglas en inglés) o análisis factorial. Sin embargo, en lugar de correlacionar las variables (como en el PCA y análisis factorial regulares) en los Q-sorts se correlacionan los participantes para dilucidar las relaciones entre ellos (Zabala, 2014).

En el año 2014, Zabala desarrolló un paquete para R (R Core Team, 2020) que mejora el software $Q$ en varias formas. En primer lugar, puede ser utilizado en distintas plataformas. En segundo lugar, permite un examen completamente transparente de las ecuaciones y las constantes utilizadas en el cálculo en cada paso del análisis, lo que ayuda a los investigadores a comprender mejor el proceso. Para la técnica de reducción de datos, también se permiten coeficientes de correlación distintos a los de Pearson. La salida de estos análisis está estructurada de manera concisa en tablas numéricas, por lo que proporciona un medio sencillo y flexible para estudiar y manejar los resultados. Por lo tanto, los resultados del método qmethod (Zabala, 2014) se pueden utilizar fácilmente para un posterior modelado cuantitativo y para la representación gráfica. Además, el resultado final de este paquete proporciona un informe más claro y sintético sobre declaraciones distintivas y de consenso. El paquete también incluye un método plot() específico 


\section{Mother Behavior Q-Set (MBQS)}

Dimensionalidad del q-set del comportamiento materno en muestras de méxico, colombia y uruguay

para crear una visualización novedosa de los resultados, así como funciones de importación y exportación (Zabala, 2014).

Los datos recopilados de todas las observaciones se introducen en una matriz con los ítems como filas y los participantes como columnas, donde los valores de las celdas son la puntuación en la cuadrícula en la que los observadores clasificaron el ítem. El proceso de análisis tiene dos partes principales. En la primera, se aplica una técnica de reducción de datos multivariante: un PCA. El algoritmo centroide para el análisis factorial es un método alternativo para el análisis factorial utilizado casi exclusivamente en la metodología Q y descrito por Brown en 1980 (Zabala, 2014).

Un componente (factor) se refiere al promedio de un Q-sort ponderado de un grupo de madres que fueron categorizadas de manera similar, y representa una perspectiva arquetípica: cómo una madre hipotética mejor representa a aquellas similares. Aunque ningún participante puede ser un representante perfecto de un factor, típicamente cada individuo es más similar a un factor que al resto (Zabala y Pascual, 2016).

Inicialmente, se construye una matriz de correlación entre los Q-sorts y la técnica multivariante elegida reduce esta matriz de correlación en componentes. Los componentes están ordenados por la variabilidad total que explican, por lo que los primeros componentes resumen la mayor parte de la variabilidad de la matriz de correlación inicial. Luego, los primeros componentes se seleccionan y se rotan para obtener una estructura clara y más simple de los datos. Los criterios habituales por los que se selecciona el número de componentes incluyen, entre otros, la cantidad total de variabilidad explicada, los eigenvalues superiores a un cierto umbral, y una solución comprometida entre la complejidad y la interpretabilidad (Zabala, 2014). La rotación de componentes en los estudios con Q-sorts puede ser manual -con base en juicios- o matemáticamente óptima -analítica- (Zabala, 2014).

La segunda parte del análisis es particular de los Q-sorts, consiste en: a) resaltar los ítems que definirán cada componente (suele nombrárseles "factor" en la literatura); b) calcular las puntuaciones de los ítems para cada factor (puntajes z y puntajes factoriales); y c) encontrar las declaraciones distintivas y de consenso. Se marcan los Q-sorts más representativos para cada factor. La marcación automática se basa en dos criterios: que la carga l debe ser significativamente alta -el umbral de significancia para un valor $p<.05$ viene dado por la ecuación 1 , donde $N$ es el número de declaraciones- (Brown, 1980 como se citó en Zabala, 2014), y que la carga cuadrada para un factor $j$ debe de ser mayor que la suma de las cargas cuadradas para todos los demás factores -ecuación 2, donde f es el número total de factores- (Zabala, 2014).

El resultado final del análisis es el número de factores seleccionados, donde cada uno representa una perspectiva. Dichas perspectivas son un Q-Sort hipotético que se ha reconstruido a partir de las puntuaciones de los factores (Zabala, 2014).

Debido a que el MBQS se ha constituido como un estándar de oro para evaluar a la sensibilidad materna y a que el proceso analítico de los Q-sorts ha recibido poca atención, el objetivo de la presente investigación fue encontrar evidencia respecto a la validez basada en la es-

tructura interna del MBQS.

\section{Método}

\section{Participantes}

Colaboraron bajo consentimiento informado distintas díadas mamá-bebé: 37 mexicanas, 35 colombianas y 24 uruguayas. Se consideraron como criterios de inclusión para las madres que su edad cronológica estuviera en el rango de los 18 a los 40 años, que no tuvieran alteraciones orgánicas y/o mentales, y que tuvieran un nivel socioeconómico medio. En el caso de los niños, que hubieran sido partos a término, sin enfermedades crónicas que alteraran su desarrollo, y con una edad cronológica comprendida entre los 5 y 45 meses de edad.

\section{Diseño}

El presente estudio - llevado a cabo en las Ciudad de México y Zona Metropolitana (México), Montevideo, Uruguay y Bogotá, Colombia- tiene un diseño de tipo transversal de alcance descriptivo.

\section{Instrumentos de recolección de datos}

El Q-Set del Comportamiento Materno, -MBQS por sus siglas en inglés-, fue diseñado por Pederson y Moran (1995a) a partir de descripciones teóricas y empíricas que toman en cuenta el constructo de sensibilidad planteado por Ainsworth et al. (1978). Consta de 90 ítems evaluados por expertos en la teoría del apego y con experiencia en la observación de la interacción mamá-bebé, quienes los utilizaron para describir a una madre prototípicamente sensible al interactuar en el hogar con su hijo de 12 meses de edad (Pederson et al., 1990). Estas descripciones fueron utilizadas para construir el criterio teórico con el que se evalúa la sensibilidad materna. La validez del instrumento se ha sustentado desde sus orígenes (e.g. Pederson et al., 1990; Pederson y Moran, 1995a).

El MBQS se aplica observando la conducta espontánea de la madre con su hijo en interacciones de la vida cotidiana. El puntaje de sensibilidad asignado a una madre corresponde al índice de correlación entre la descripción del comportamiento materno y el criterio de descripción teórico, reflejando similitudes o diferencias con el perfil de la madre prototípicamente sensible (-1 a 1). Para el presente estudio utilizamos la traducción al castellano de Posada y equipo (2002).

En cuanto a la confiabilidad de las sub-escalas del MBQS propuestas por Posada et al. (2002), se reportó una consistencia de $\alpha=0.92$ para Respuesta sensible; Accesibilidad de $\alpha=0.88$; Aceptación del bebé de $\alpha=0.83$; Interferencia de $\alpha=0.76$; Activa-Animada de $\alpha=0.89$; Crea un ambiente interesante de $\alpha=0.80, \mathrm{y}$; Preocupación por apariencia física obtuvo una confiabilidad de $\alpha=0.73$ (Posada et al., 2002).

\section{Procedimiento}

Para la aplicación del MBQS es necesario un entrenamiento especializado, el cual fue dirigido y supervisado en México por el primer autor, en Colombia por la tercera autora, y en Uruguay por la cuarta y quinta autoras, quienes a su vez fueron entrenados por Germán Posada y/o Olga Alicia Carbonell, especialistas en los Q-sorts y en el MBQS. Para certificarse, se requiere obtener puntajes $\geq .80$ de confiabilidad inter-observadores en los videos de entrenamiento. Para considerar a 
cada uno de los tres equipos preparados para realizar observaciones, empleamos el mismo criterio; es decir, sólo salieron al campo los observadores que obtuvieron $\geq .80$ de confiabilidad inter-observadores en los videos de entrenamiento correspondientes.

El entrenamiento en el uso del MBQS consistió en cuatro sesiones, dando un total de por lo menos 14 horas de preparación. Los entrenamientos tuvieron como prerrequisitos distintas experiencias en los tres países: primero se dieron las bases teóricas de la teoría del apego; en segundo lugar, se presentó formalmente el proyecto de investigación, y por último; se profundizó en los Q-sorts. La preparación propiamente consistió en la lectura y discusión del significado de los 90 ítems, para posteriormente practicar la observación y ordenamiento gracias al apoyo de al menos cuatro videos de interacciones madre-bebé en visitas domiciliarias. Las descripciones de los observadores se compararon con puntajes ofrecidos por expertos. En el entrenamiento, los índices de confiabilidad inter-observador (calculados con base en el acuerdo entre las descripciones de dos observadores independientes) variaron entre .81 y .96 para el equipo mexicano; .72 a .97 para el equipo uruguayo y .77 a .96 para el equipo colombiano.

Después del contacto inicial con cada madre, se realizó una visita para observarles en interacción directa con sus hijos. Tales observaciones, de entre 1.5 y 2 horas de duración, fueron videograbadas para que posteriormente dos observadores independientes y debidamente entrenados en el MBQS evaluaran el comportamiento materno (i.e. la sensibilidad). La confiabilidad inter-observador para las 37 observaciones realizadas en México fue de .88 (rango = .83 a .93); para las 35 observaciones llevadas a cabo en Colombia fue de .86 ( rango = .75 a .96), y para las 24 observaciones uruguayas fue de .91 (rango $=.72$ a .97).

\section{Estrategia para el análisis de datos}

Se obtuvieron estadísticos descriptivos de la variable continua de la sensibilidad; esto es, del puntaje global del MBQS: Se realizó un análisis factorial Q para establecer los perfiles de madres en los tres países; es decir, un análisis para determinar el número de factores a conservar. Con respecto a estos perfiles (factores), se reporta su confiabilidad, valores Eigen, porcentaje de varianza explicada y confiabilidad compuesta (Zabala, 2014). Asimismo, con el fin de comparar los resultados de esta muestra con los reportados por Posada y colaboradores (2002) se realizó un análisis factorial confirmatorio cuyo ajuste se determina mediante los coeficientes ji cuadrada y los índices de ajuste RMSEA, CFI y TLI (Hanckock y Mueller, 2010). Todos los análisis se llevaron a cabo en el ambiente estadístico R Core Team (2017) versión 3.4.3.

\section{Consideraciones éticas}

Se informó a las madres de familia que su participación en este estudio era confidencial y proporcionaron su consentimiento verbal y escrito antes de participar. Todos los procedimientos realizados estuvieron de acuerdo con los estándares éticos de los comités de investigación institucionales de los tres países, así como con la declaración de Helsinki de 1964 y sus posteriores modificaciones o estándares éticos comparables. El Comité de Ética de la universidad de adscripción la cuatra y quinta autoras aprobó el proyecto con fecha del 27 de septiembre del 2013.

\section{Resultados}

Todas las díadas tenían un nivel socioeconómico medio según las clasificaciones utilizadas en Colombia, México y Uruguay. A excepción de dos mujeres mexicanas y una colombiana, todas las participantes tenían, por lo menos, educación secundaria. La edad promedio para las madres mexicanas fue de 29.32 años ( $D E=10.14$ ) y para los hijos 2.27 años ( $D E=1.64$ ), siendo 17 niñas y 20 niños. Las mamás colombianas tenían en promedio 27.26 años de edad ( $D E=4.79$ ) y sus hijos 4.97 meses $(D E=0.34)$ : participaron 14 niñas y 21 niños. Por último, la edad promedio para las madres uruguayas fue de 33.33 años ( $D E=4.11) \mathrm{y}$ para sus hijos 11.42 meses ( $D E=4.11)$, siendo 11 niños y 13 niñas.

En la tabla 1 se muestran medidas de tendencia central y de dispersión para los puntajes globales del MBQS en los tres países.

Tabla 1. Puntajes globales del MBQS

\begin{tabular}{cccc}
\hline MBQS & Rango & Media & DE \\
\hline Colombia & $0.5-0.85$ & 0.69 & 0.21 \\
\hline México & $-0.44-0.84$ & 0.57 & 0.37 \\
\hline Uruguay & $0.60-0.86$ & 0.78 & 0.07 \\
\hline
\end{tabular}

Fuente: elaboración propia

Posteriormente, se llevó a cabo un análisis factorial (qmethod, Zabala, 2014) que arrojó tres factores o grupos de madres de acuerdo con sus puntuaciones: 1) madres sumamente sensibles; 2) madres sensibles y 3) madres no sensibles. Cabe señalar que el tercer grupo se conformó únicamente por una madre que presentó respuestas totalmente contrarias a los criterios teóricos (i.e., una madre insensible). Ver Tabla 2

Tabla 2. Características de los factores para el modelo de análisis factorial $Q$ y cargas factoriales

\begin{tabular}{ccccccc}
\hline $\begin{array}{c}\text { Perfil } \\
\text { (factor) }\end{array}$ & $\begin{array}{c}\text { Promedio } \\
\text { confiabilidad }\end{array}$ & N & $\begin{array}{c}\text { Valores } \\
\text { eigen }\end{array}$ & $\begin{array}{c}\text { Var. } \\
\text { exp. }\end{array}$ & $\begin{array}{c}\text { Confiabilidad } \\
\text { compuesta }\end{array}$ & $\begin{array}{c}\text { EEM } \\
\text { puntajes } \\
\text { de factor }\end{array}$ \\
\hline $\begin{array}{c}\text { Sumamente } \\
\text { sensibles }\end{array}$ & 0.8 & 88 & 62.392 & 64.321 & 0.997 & 0.053 \\
\hline Sensibles & 0.8 & 6 & 3.680 & 3.794 & 0.960 & 0.200 \\
\hline Insensibles & 0.8 & 1 & 3.123 & 3.219 & 0.800 & 0.447 \\
\hline
\end{tabular}

Fuente: elaboración propia

Se llevaron a cabo los modelos de análisis factorial confirmatorio con los grupos 1 y 2 (madres sumamente sensibles y madres sensibles). Para el análisis confirmatorio de siete factores correlacionados (las siete escalas encontradas para la muestra colombiana de Posada et al. 2002), se probaron soluciones (ejes principales, factores principales, etc.) y estimadores (WLS, DWLS, etc.); empero, en ninguna de ellas se obtuvo un buen ajuste $(=1943.012,<.0001 ;=0.107[0.100,0.114]$; = $0.473 ;=0.44$ ). Pese a esto, las medias de puntaje por ítem para el grupo de madres sensibles son congruentes con las reportadas en dicha investigación con una diferencia máxima de 2.46 (ver Tabla 3).

Tabla 3. Comparación de la media por ítem para el grupo de madres sensibles contra los puntajes criterio (Posada et al., 2002)

\begin{tabular}{|cccc|}
\hline İtem & Criterio & Media & Diferencia \\
\hline MBQS1 & 8.00 & 7.69 & 0.31 \\
\hline MBQS2R & 1.00 & 2.55 & -1.55 \\
\hline MBQS3 & 1.92 & 3.06 & -1.14 \\
\hline MBQS4R & 1.33 & 2.15 & -0.82 \\
\hline MBQS5 & 7.92 & 7.56 & 0.36 \\
\hline MBQS6 & 8.50 & 7.30 & 1.20 \\
\hline
\end{tabular}


Mother Behavior Q-Set (MBQS)

Dimensionalidad del q-set del comportamiento materno en muestras de méxico, colombia y uruguay

\begin{tabular}{|c|c|c|c|}
\hline Ítem & Criterio & Media & Diferencia \\
\hline MBQS7R & 1.25 & 2.55 & -1.30 \\
\hline MBQS8R & 1.33 & 2.07 & -0.74 \\
\hline MBQS9 & 8.92 & 7.92 & 1.00 \\
\hline MBQS10 & 7.25 & 4.79 & 2.46 \\
\hline MBQS11R & 2.25 & 3.43 & -1.18 \\
\hline MBQS12 & 9.00 & 7.58 & 1.42 \\
\hline MBQS13R & 2.75 & 4.15 & -1.40 \\
\hline MBQS14 & 4.08 & 3.69 & 0.39 \\
\hline MBQS15 & 7.58 & 5.47 & 2.11 \\
\hline MBQS16 & 2.00 & 2.82 & -0.82 \\
\hline MBQS17 & 3.92 & 4.06 & -0.14 \\
\hline MBQS18 & 7.17 & 7.13 & 0.04 \\
\hline MBQS19 & 2.58 & 3.56 & -0.98 \\
\hline MBQS20R & 2.50 & 2.94 & -0.44 \\
\hline MBQS21 & 5.75 & 7.04 & -1.29 \\
\hline MBQS22 & 6.83 & 5.66 & 1.17 \\
\hline MBQS23 & 7.67 & 7.51 & 0.16 \\
\hline MBQS24 & 7.17 & 6.73 & 0.44 \\
\hline MBQS25 & 4.25 & 3.45 & 0.80 \\
\hline MBQS26 & 3.50 & 3.96 & -0.46 \\
\hline MBQS27R & 4.67 & 3.04 & 1.63 \\
\hline MBQS28R & 1.67 & 2.39 & -0.72 \\
\hline MBQS29 & 8.83 & 7.27 & 1.56 \\
\hline MBQS30 & 5.67 & 6.29 & -0.62 \\
\hline MBQS31 & 5.58 & 5.82 & -0.24 \\
\hline MBQS32 & 5.83 & 7.17 & -1.34 \\
\hline MBQS33 & 6.00 & 7.00 & -1.00 \\
\hline MBQS34 & 7.00 & 7.61 & -0.61 \\
\hline MBQS35 & 6.17 & 6.39 & -0.22 \\
\hline MBQS36 & 6.75 & 7.71 & -0.96 \\
\hline MBQS37 & 6.25 & 6.50 & -0.25 \\
\hline MBQS38 & 6.50 & 7.57 & -1.07 \\
\hline MBQS39 & 6.00 & 7.51 & -1.51 \\
\hline MBQS40 & 6.75 & 6.24 & 0.51 \\
\hline MBQS41R & 3.67 & 1.94 & 1.73 \\
\hline MBQS42 & 6.92 & 7.53 & -0.61 \\
\hline MBQS43 & 4.25 & 6.34 & -2.09 \\
\hline MBQS44 & 7.42 & 5.68 & 1.74 \\
\hline MBQS45 & 7.58 & 6.26 & 1.32 \\
\hline MBQS46 & 8.17 & 6.44 & 1.73 \\
\hline MBQS47 & 7.67 & 6.58 & 1.09 \\
\hline MBQS48 & 5.08 & 6.49 & -1.41 \\
\hline MBQS49 & 6.17 & 6.07 & 0.10 \\
\hline MBQS50 & 3.75 & 3.38 & 0.37 \\
\hline MBQS51 & 3.58 & 3.62 & -0.04 \\
\hline MBQS52 & 1.67 & 3.84 & -2.17 \\
\hline MBQS53 & 8.58 & 7.15 & 1.43 \\
\hline MBQS54 & 9.00 & 7.12 & 1.88 \\
\hline MBQS55 & 2.42 & 4.88 & -2.46 \\
\hline MBQS56 & 4.33 & 3.90 & 0.43 \\
\hline MBQS57R & 1.25 & 2.45 & -1.20 \\
\hline MBQS58 & 8.08 & 6.87 & 1.21 \\
\hline MBQS59R & 1.75 & 2.27 & -0.52 \\
\hline MBQS60 & 8.75 & 6.98 & 1.77 \\
\hline MBQS61 & 8.08 & 6.20 & 1.88 \\
\hline MBQS62R & 2.17 & 3.31 & -1.14 \\
\hline MBQS63 & 8.83 & 7.25 & 1.58 \\
\hline MBQS64 & 8.17 & 6.76 & 1.41 \\
\hline
\end{tabular}

\begin{tabular}{|c|c|c|c|}
\hline Ítem & Criterio & Media & Diferencia \\
\hline MBQS65R & 2.58 & 2.99 & -0.41 \\
\hline MBQS66 & 8.25 & 7.33 & 0.92 \\
\hline MBQS67 & 7.67 & 7.83 & -0.16 \\
\hline MBQS68R & 1.42 & 2.56 & -1.14 \\
\hline MBQS69 & 3.92 & 3.09 & 0.83 \\
\hline MBQS70 & 3.33 & 3.23 & 0.10 \\
\hline MBQS71 & 2.08 & 3.43 & -1.35 \\
\hline MBQS72R & 4.33 & 3.57 & 0.76 \\
\hline MBQS73 & 1.50 & 3.32 & -1.82 \\
\hline MBQS74 & 1.58 & 3.05 & -1.47 \\
\hline MBQS75 & 3.83 & 2.61 & 1.22 \\
\hline MBQS76R & 3.50 & 3.64 & -0.14 \\
\hline MBQS77 & 3.42 & 4.40 & -0.98 \\
\hline MBQS78 & 2.42 & 3.93 & -1.51 \\
\hline MBQS79 & 5.58 & 5.87 & -0.29 \\
\hline MBQS80R & 2.67 & 2.06 & 0.61 \\
\hline MBQS81 & 4.25 & 4.53 & -0.28 \\
\hline MBQS82 & 5.50 & 4.87 & 0.63 \\
\hline MBQS83R & 3.50 & 5.26 & -1.76 \\
\hline MBQS84 & 2.67 & 2.10 & 0.57 \\
\hline MBQS85 & 5.08 & 4.87 & 0.21 \\
\hline MBQS86 & 6.25 & 6.14 & 0.11 \\
\hline MBQS87 & 3.08 & 1.67 & 1.41 \\
\hline MBQS88R & 2.33 & 2.94 & -0.61 \\
\hline MBQS89 & 5.75 & 4.85 & 0.90 \\
\hline MBQS90 & 6.08 & 6.85 & -0.77 \\
\hline
\end{tabular}

Ítem

MBQS8R

MBQS10

MBQS12

$M B Q S 14$

MBQS15

MBQS17

MBQS19

MBQS21

MBQS23

MBQS25

MBQS26

MBQS28R

MBQS31

MBQS33

MBQS35

MBOS38

MBOS40

MBQS41R

MBQS43

MBQS44

MBQS47

MBQS49

MBQS50

MBOS52

MBQS53

MBQS55

MBQS56

MBQS59R

MBQS62

MBQS64

Se realizaron análisis unifactoriales para cada escala obteniendo un buen ajuste al modelo en las escalas de Interferencia, Crea un ambiente interesante y Preocupada por la apariencia física (Ver Tabla 4).

Tabla 4. Medidas de ajuste para los modelos unidimensionales de las siete escalas reportadas en Posada et al. (2002)

\begin{tabular}{cccccccc}
\hline $\begin{array}{c}\text { Escala del } \\
\text { MBQS }\end{array}$ & chisq & Df & Cfi & Rmsea & $\begin{array}{c}\text { rmsea. } \\
\text { ci.lower }\end{array}$ & $\begin{array}{c}\text { rmsea. } \\
\text { ci.upper }\end{array}$ & Srmr \\
\hline $\begin{array}{c}\text { Respuesta } \\
\text { Sensible }\end{array}$ & 171.990 & 54 & 0.607 & 0.158 & 0.131 & 0.184 & 0.107 \\
\hline Accesibilidad & 98.994 & 27 & 0.692 & 0.174 & 0.138 & 0.212 & 0.125 \\
\hline $\begin{array}{c}\text { Aceptación del } \\
\text { bebé }\end{array}$ & 30.747 & 9 & 0.836 & 0.166 & 0.104 & 0.232 & 0.098 \\
\hline $\begin{array}{c}\text { Interferencia } \\
\text { Activa-Animada }\end{array}$ & 0.000 & 0 & 0.998 & 0.000 & 0.000 & 0.000 & 0.000 \\
\hline $\begin{array}{c}\text { Crea un ambiente } \\
\text { interesante }\end{array}$ & 0.000 & 0 & 0.998 & 0.000 & 0.000 & 0.000 & 0.000 \\
$\begin{array}{c}\text { Preocupación por } \\
\text { la apariencia física }\end{array}$ & 0.000 & 0 & 0.998 & 0.000 & 0.000 & 0.000 & 0.000 \\
\hline
\end{tabular}

Fuente: elaboración propia

\section{Discusión y Conclusiones}

En el presente estudio llevamos a cabo dos metodologías con diferentes enfoques: el análisis de factor Q, que permite identificar subconjuntos de individuos en una muestra, y el análisis factorial confirmatorio con un enfoque cuantitativo tradicional, que analiza las covarianzas de los ítems para disminuir el número de dimensiones en un instrumento de medición y que, al ser un análisis paramétrico, requiere del cumplimiento de ciertos supuestos en cuanto al número de observaciones y parámetros a estimar. 
En la investigación descrita en el primer apartado del documento, realizada por Posada y colaboradores en el año 2002, se reportan datos sobre la validez del MBQS con base en su estructura interna, lo que de forma holística permitió que dicho equipo construyera escalas interpretables. Bajo el análisis factorial confirmatorio, las escalas propuestas por dicho equipo de investigación en las que nuestro modelo factorial presentó un buen ajuste son las que tienen menos ítems -menos parámetros por estimar-, lo cual puede ser indicativo de que la falta de ajuste tiene que ver con la identificación del modelo debido a que contamos con una muestra limitada, tanto de madres (88) como de ítems del MBQS (46).

Por lo anteriormente descrito, una limitación del presente estudio es el tamaño de la muestra, ya que cabe la posibilidad de que con una suficientemente numerosa, las otras escalas y el modelo completo de siete factores presente un buen ajuste. Sería necesario realizar más pruebas con muestras de mayor tamaño para comprobar esta hipótesis.

No es nuestro propósito desvalorizar la propuesta de Posada et al. (2002), ya que las escalas conformadas con base en su trabajo han sido utilizadas en otras investigaciones que han reportado asociaciones importantes entre los puntajes de las escalas del MBQS y características de las madres. No obstante, estas escalas se conformaron de una mezcla de fuentes de información entre el PCA, la correlación de los ítems con la puntuación de la escala y las cualidades de contenido de los reactivos, por lo que la falta de ajuste al modelo factorial confirmatorio era esperable. De acuerdo con lo planteado por Block (1978), esta falta de ajuste puede deberse al limitado número de observaciones, lo cual es congruente con nuestros resultados.

Independientemente de este hallazgo, la presente pesquisa es relevante porque: a) retoma la propuesta del doctor Germán Posada y sus colaboradores -nuestro profesor y formador, experto en teoría del apego, amplia e internacionalmente reconocido, así como pionero en la realización de pesquisas conducidas desde este marco teórico en América Latina-; b) utiliza el MBQS, considerado uno de los estándares de oro para la evaluación del comportamiento materno -comunmente reducido a la sensibilidad-, el cual ha sido poco empleado en nuestra región; c) cuenta con muestras de tres países latinoamericanos, lo cual fue posible gracias al espíritu colaborativo de la Red Iberoamericana de Apego, tal y como su fundador y nuestro querido maestro L. Alan Sroufe lo conceptualizó; d) pone atención al proceso analítico y hace uso de los recientes avances estadísticos y computacionales, lo cual es sumamente novedoso, y; e) hecha mano de un software libre y de un paquete sencillo de utilizar, lo cual permite que cualquier persona con acceso a internet pueda emplearlo, así como encontrar ayuda y tutoriales en línea sin costo.

¿Qué se desprende para investigaciones futuras? Replicar resultados en otros países y comparar puntuaciones de los grupos de madres sensibles; conseguir más participantes o juntar muestras para lograr un número de observaciones apropiado para correr el modelo de factorial confirmatorio, además de poder realizar un análisis factorial para analizar diferencias entre grupos (países, estados, etc.).

Pese a lo anterior, consideramos que tanto el uso del MBQS para evaluar el comportamiento de las madres colombianas, uruguayas y mexicanas, como del paquete "qmethod" para realizar el análisis de los datos resulta novedoso, relevante y pertinente.

\section{Referencias}

Ainsworth, M.D.S. (1967). Infancy in Uganda: Infant care and the growth of love. Baltimore: John Hopkins University Press. https://psycnet.apa. org/record/1967-35025-000

Ainsworth, M.D.S. (1973). Systems for rating maternal care behaviors. En E.G. Boyer, A. Simon, G. Karafin, y R. Karafin (Eds.), Measures of maturation: An anthology of early childhood observation instruments (Vol. 1; pp. 67-172). Philadelphia, USA: Research for Better Schools, Inc. https://doi.org/10.1016/S2007-4719(14)70396-5

Ainsworth, M. D. S., Bell, S. M., y Stayton, D. J. (1974). Infant-mother attachment and social development: "Socialization" as a product of reciprocal responsiveness to signals. In P. Richards (Ed.), The integration of a child into a social word (pp.99-135). Cambridge: Cambridge University Press. https://psycnet.apa.org/ record/1975-07118-004

Ainsworth, M. D. S., Blehar, M. C., Waters, E., y Wall, S. (1978). Patterns of attachment. Hillsdale, NJ: Erlbaum. https://mindsplain.com/wpcontent/uploads/2021/01/Ainsworth-Patterns-of-Attachment.pdf

Atkinson, L., Niccols, A., Paglia, A., Coolbear, J., Parker, K., Poulton, L., Guger, S. y Sitarenious, G. (2000). A meta-analysis of time between maternal sensitivity and attachment assessments: Implications for internal working models in infancy toddlerhood. Journal of Social and Personal Relationships, 17(6), 791-810.dol:10.1177/0265407500176005

Bakermans-Kranenburg, M. J., van IJzendoorn, M. H., y Juffer, F. (2003). Less is more: Meta-analyses of sensitivity and attachment interventions in early childhood. Psychological Bulletin, 129, 195-215. doi:10.1037/0033-2909.129.2.195

Block, J. (1978). The q-sort method. Palo Alto, CA: Consulting Psychologist Press.http://www.psychology.sunysb.edu/attachment/measures/ content/Jack_Block_Q-sort_method_book.pdf

Bowlby, J. (1969/1982). Attachment and loss (Vol. I): Attachment. New York, NY: Basic Books.https://mindsplain.com/wpcontent/uploads/2020/08/ ATTACHMENT AND LOSS VOLUME I ATTACHMENT.Pdf

Bowlby, J. (1988). A secure base. New York: Basic Books. https://books. google.es/books?hl=es\&lr=\&id=yA9nX8W2ddIC\&oi=fnd\&pg=PP2\&dq=Bowlby, +J.+(1988).+A+secure+base.+New+York:+Basic+Books. + \&ots $=1 \mathrm{kmH}$ HuW $9 \mathrm{w}$ v\&sig=0PbjoD kwQon2kRxwgXTbMtgYRRc\#v=onepage \&q=Bowlby\%2C\%20 J.\%20(1988).\%20A\%20secure\%20 base.\%20New\%20York\%3A\%20Basic\%20Books.\&f=false

Cadman, T., Diamond, P., y Fearon, P. (2017). Reassessing the validity of the attachment Q-sort: An updated meta-analysis. Inf Child Dev. doi:10.1002/icd.2034

Carbonell, O.A., Plata, S.J. y Alzate, G. (2006). Creencias y expectativas sobre el comportamiento materno ideal y real en mujeres gestantes desde un abordaje metodológico mixto. Revista Infancia, Adolescencia y Familia, 1(001), 115-140. https://www. researchgate.net/profile/Olga-Carbonell/publication/237612113 CREENCIAS Y EXPECTATIVAS SOBRE EL COMPORTAMIENTO MATERNO_IDEAL_Y_REAL_EN_MUJERES_GESTANTES_DESDE_UN_ ABORDAJE METODOLOGICO MIXTO/links/54e39fd80cf2b2314f5e0147// CREENCIAS-Y-EXPECTATIVAS-SOBRE-EL-COMPORTAMIENTO-MATERNOIDEAL-Y-REAL-EN-MUJERES-GESTANTES-DESDE-UN-ABORDAJEMETODOLOGICO-MIXTO.pdf

De Wolff, M. S. y van IJzendoorn, M. H. (1997). Sensitivity and attachment: A meta-analysis on parental antecedents of infant attachment. Child Development, 68, 571-591. doi:10.2307/1132107

Duarte-Rico, L., García Ramírez, N., Rodríguez-Cruz, E., y Bermúdez-Jaimes, M. (2016). Las prácticas de crianza y su relación con el vínculo afectivo. Revista Iberoamericana de Psicología: Ciencia y Tecnología 9(2), 113-124. http://orcid.org/0000-0002-5049-7073

Fourment, K., Nóblega, M., Conde, G., Nuñez del Prado, J., y Mesman, J (2020). Maternal sensitivity in rural Andean and Amazonian Peru. Attachment \& Human Development. doi:10.1080/14616734.2020.1828 $\underline{515}$ 


\section{Mother Behavior Q-Set (MBQS)}

\section{Dimensionalidad del q-set del comportamiento materno en muestras de méxico, colombia y uruguay}

Hancock, G. R. y Mueller, R. O. (Eds.). (2010). The reviewer's guide to quantitative methods in the social sciences. New York: Routledge. https:// books.google.es/books? hl=es\&lr=\&id=8zz3DWAAQBAJ\&oi=fnd\&pg $=P$ P1\&dq=Hancock,$+G .+R .+y+$ Mueller, + R. + O. $+($ Eds. $) .+(2010) .+$ The + reviewer\%27s+quide+to+quantitative+methods + in + the + social + sciences. +New+York:+Routledge.\&ots=pNeCO35MZI\&sig=njos-oZvHH6pwVInTGu RUSPgGV8\#V=Onepage\&q=Hancock\%2C\%20G.\%20R.\%20y\%20 Mueller\%2C\%20R.\%200.\%20(Eds.).\%20(2010).\%20The\%20reviewer's\%20quide\%20to\%20quantitative\%20methods\%20in\%20the\%20 social\%20sciences.\%20New\%20York\%3A\%20Routledge.\&f=false

Nievar, M. y Becker, B. (2008). Sensitivity as a privileged predictor of attachment: a second perspective on De Wolff and van ljzendoorn's Meta-analysis. Social Development, 17, 102-114. doi:10.1111/j.14679507.2007.00417.x

Pederson. D. R. y Moran, G. (1995a). A categorical description of infantmother relationships in the home and its relation to Q- sort measures of infant-mother interaction. En E. Waters, B. E. Vaughn, G. Posada, y K. Kondo-Ikemura (Eds.), Caregiving, cultural, and cognitive perspectives on secure-base behavior and working models: New growing points of attachment theory and research. Monographs of the Society for Research in Child Development, 60 (2-3), 111-132. https://doi.org/10.1111/j.1540-5834.1995.tb00207.x

Pederson, D. R., y Moran, G. (1995b). Appendix B. Maternal Behavior Q-set. En E. Waters, B. E. Vaughn, G. Posada, y K. Kondo-Ikemura (Eds.) Caregiving, cultural, and cognitive perspectives on secure-base behavior and working models: New growing points of attachment theory and research. Monographs of the Society for Research in Child Development 60 (2-3), 247-254. DOI: 10.1016/0378-3782 (96) 88182-5

Pederson D.R., Moran, G., Sitko, C., Campbell, Ghesquire, K., y Acton, H. (1990). Maternal Sensitivity and the Security of Infant-Mother Attachment: A Q-Sort Study. Child Development. 61 (6), 1974-1983. https://doi.org/10.1111/j.1467-8624.1990.tb03579.x

Posada, G. y Waters, E. (2014). El sistema de comportamiento de cuidado: Sensibilidad y apoyo de base segura. En B. Torres, J. Causadias, y G. Posada (Eds.), La teoría del apego: Investigación y aplicaciones clínicas (pp. 75-97). Madrid: Psimática Editorial, S.L.

Posada, G. y Waters, H.S. (2018). Introduction: The co-construction of mother-child attachment relationships in early childhood. In G. Posada and H.S. Waters (Eds.), The mother-child attachment partnership in early childhood: secure base behavioral and representational processes. Monographs of the Society for Research in Child Development, 83: 7-21. doi:10.1111/mono.12388

Posada, G., Carbonell, O.A., Alzate, G., y Plata, S.J. (2004). Through Colombian lenses: Ethnographic and conventional analyses of maternal care and their association with secure base behavior. Developmental Psychology, 40, 508-518. doi:10.1037/0012$\underline{1649.40 .4 .508}$
Posada, G., Jacobs, A., Richmond, M. K., Carbonell, O. A., Alzate, G., Bustamante, M. R., y Quiceno, J. (2002). Maternal caregiving and infant security in two cultures. Developmental Psychology, 38, 67-78. doi:10.1037//0012-1649.38.1.67

Posada, G., Trumbell, J., Nóblega, M., Plata, S.J., Peña, P., Carbonell, O.A., et al., (2016). Maternal sensitivity and child secure base use in early childhood: Studies in different cultural contexts. Child Development, 87, 297-311. doi:10.1111/cdev.12454

R Core Team. (2017). R: A language and environment for statistical computing (versión 3.4.3) [software de cómputo]. Vienna, Austria. Recuperado de https://www.R-project.org/

Salinas-Quiroz, F., y Posada, G. (2015). MBQS: Método de evaluación para intervenciones en apego dirigidas a primera infancia. Revista Latinoamericana de Ciencias Sociales, Niñez y Juventud, 13(2), 10511063. doi:10.11600/1692715x.13235280514

Salman-Engin, S., Aran, Ö., Karagöbek, A.B., y Sümer, N. (2017 June 29 July 1st). Caregiving Behavior Profiles among Turkish Mothers. The International Attachment Conference 2017, London, United Kingdom.

van IJzendoorn, M.H. y Sagi-Schwartz, A. (2008). Cross-cultural patterns of Attachment. Universal and contextual dimensions. In J. Cassidy y P.R. Shaver (Eds.), Handbook of attachment. Theory, research and the clinical applications (pp.880-905). New York, NY: Guilford Press. https://psycnet.apa.org/record/2008-13837-038

Verhage, M. L., Schuengel, C., Madigan, S., Fearon, R. M. P., Oosterman, M., Cassibba, R., Bakermans-Kranenburg, M.J., y van IJzendoorn, M. H., (2016). Narrowing the transmission gap: A synthesis of three decades of research on intergenerational transmission of attachment. Psychological Bulletin, 142, 337-366. doi: 10.1037/bul0000038

Zabala, A. (2014). qmethod: A Package to Explore Human Perspectives Using Q Methodology. The R Journal, 6 (2), 163-173.http://journal.rproject.org/archive/2014-2/zabala.pdf

Zabala, A y Pascual, U. (2016). Bootstrapping Q Methodology to Improve the Understanding of Human Perspectives. PLoS ONE, 11(2): e0148087. doi: 10.1371/journal.pone.0148087

Zeegers, M.A.J., Colonnesi, C., Stams, G.J.M., y Meins, E. (2017). Mind matters: A meta-analysis on parental mentalization and sensitivity as predictors of infant-parent attachment. Psychol Bull, 143, 1245-1272. doi:10.1037/bul0000114 